\title{
Themenschwerpunkt
}

Tobias Dürr*

\section{Regieren ohne Grund: Die CDU mit und nach Merkel}

https://doi.org/10.1515/fjsb-2021-0037

Zusammenfassung: Von 72 Jahren seit der Gründung der Bundesrepublik im Jahr 1949 stellte die Christdemokraten 52 Jahre den Bundeskanzler oder die Bundeskanzlerin. Vielleicht wird dies auch nach den Bundestagswahlen 2021 so sein. Dennoch bleibt zu fragen, ob mit Armin Laschet die CDU einen zeitgemäßen Vorsitzenden und potentiellen Bundeskanzler stellt, der die anstehenden großen Herausforderungen etwa in der Klimapolitik angemessen beantworten kann.

Summary: Out of the 72 years since the founding of the Federal Republic in 1949, the Christian Democrats have provided the Federal Chancellor - male or female for 52 years. Perhaps this will also be the case after the 2021 federal elections. Nevertheless, the question remains whether the CDU has found a contemporary chairman and potential chancellor in Armin Laschet, one who can adequately respond to the major challenges ahead such as, for instance, climate policy.

„Es geht alles schief, es geht alles schief“, ruft Wolfgang Schäuble entsetzt aus. Als der entfesselte Machtkampf um die Kanzlerkandidatur der Unionsparteien zwischen Armin Laschet und Markus Söder in der Nacht zum 20. April 2021 seinem dramatischen Finale entgegenschlingert, bekommt es selbst der große alte Mann der deutschen Christdemokratie mit der Angst zu tun. Die entscheidende Vorstandssitzung der CDU droht zu scheitern. Nur vordergründig bezieht sich Schäubles Panik auf das digitale Unvermögen etlicher Sitzungsteilnehmer. In Wirklichkeit gilt sie in allumfassender Weise der Verfassung seiner Partei insgesamt. Diese beschreibt Schäuble als ,jammervoll“. Am Ende der Nacht setzt er seinen Favoriten fast im Alleingang durch. Dabei unterstützt Schäuble Armin Laschet im Grunde nur, weil er Markus Söder verhindern will - und damit eine Art feindliche Übernahme der CDU durch die CSU. So wird Armin Laschet Kanzlerkandidat der Unionsparteien. Nicht ausgeschlossen, dass CDU und CSU mit ihm an der Spitze am 26. September noch einmal als erste durchs Ziel gehen. Und

*Kontakt: Dr. Tobias Dürr, Politikwissenschaftler, Publizist, Buchautor, Redenschreiber und Politikberater. 
trotzdem ist für die CDU tatsächlich so gut wie alles schiefgegangen. Nicht erst in dieser Nacht. Sondern seit vielen Jahren schon.

Wer das Ausmaß der Misere ermessen will, muss sich noch einmal grundsätzlich vor Augen führen, aus welchen Höhen die deutsche Christdemokratie herabgesunken ist. Die CDU hat die Bundesrepublik politisch geprägt, dominiert und politisch verortet wie keine andere Partei. Es waren christdemokratische Kanzler, die alle Fundamentalfestlegungen der Republik durchsetzten, von der Westbindung (Adenauer) über die soziale Marktwirtschaft (Erhard) bis hin zu europäischer Einigung und deutscher Einheit (Kohl).

In den 72 Jahren seit der Gründung der Bundesrepublik im Jahr 1949 standen Christdemokrat*innen über 52 Jahre hinweg an der Spitze der Bundesregierung. Gerade einmal 20 Jahre lang befand sich das Kanzleramt nicht in christdemokratischen Händen. Zweimal bereits in der Geschichte der Bundesrepublik sprach einiges dafür, dass es um die strukturelle Vorherrschaft der Christdemokratie endgültig geschehen sein könnte. Doch anders als von vielen Zeitgenossen jeweils erwartet, führte weder der „Machtwechsel“ von 1969 noch der rot-grüne „Aufbruch“ von 1998 zur grundlegenden Abkehr vom alten Grundmuster christdemokratischer Dominanz. Die ewige Machtmaschine CDU rollte immer weiter, bis heute - so scheint es jedenfalls.

Währte die sozialliberale Ära Brandt/Schmidt/Genscher insgesamt immerhin 13 Jahre, blieben dem rot-grünen „Aufbruch“ unter Gerhard Schröder und Joschka Fischer nicht einmal zwei volle Wahlperioden beschieden. Beide Male folgte auf die Abweichung von der Norm - wenigstens oberflächlich betrachtet - die Rückkehr zum früheren Normalmaß unter christdemokratischer Führung. Allein die beiden jeweils 16 Jahre währenden Kanzlerschaften Kohl und Merkel entsprechen in ihrer Dauer zusammengerechnet nicht sehr viel weniger als der Hälfte des gesamten bisherigen Bestehens der Bundesrepublik. Und selbst als die Union zwischendurch zweimal die Macht in der Republik einbüßte, blieb sie im Vergleich zur zweiten großen Volkspartei der Bundesrepublik doch fast immer die stärkere, gesellschaftlich breiter und tiefer verwurzelte politische Kraft. Das war so beim Machtwechsel von 1969, als die Unionsparteien mit einem überlegenen Wahlergebnis von 46,1 Prozent (gegenüber 42,7 Prozent für die SPD) als gefühlter Wahlsieger den Gang in die Opposition antraten. Und das war erst recht so im Fall der „Wahlniederlage“ von 1976, als die Union unter Führung von Helmut Kohl volle 48,6 Prozent der Stimmen für sich verbuchen konnte (gegenüber 42,6 Prozent für die SPD) - das zweithöchste Unionsergebnis seit 1949. Überhaupt nur bei zwei der bislang 19 Wahlen zum Deutschen Bundestag, nämlich 1972 und dann nochmals 1998, gingen die Unionsparteien hinter ihrer sozialdemokratischen Konkurrenz durchs Ziel. Abgerundet wurde das Bild umfassender christdemokratischer Dominanz durch triumphale Wahlergebnisse in fast allen Flächenländern der Repu- 
blik - oft deutlich oberhalb von 50 Prozent, so etwa in Rheinland-Pfalz (dreimal zwischen 1971 und 1979), Baden-Württemberg (viermal zwischen 1972 und 1984), Schleswig-Holstein (1971) oder Niedersachsen (1980). Parallel dazu versammelte die CSU als bayerische Schwesterpartei der CDU zwischen 1970 und 2008 sogar neunmal in Folge absolute Wählermehrheiten hinter sich.

Ohne Wenn und Aber die Hegemonialpartei der alten Bundesrepublik war die CDU, weil sie sich wie keine andere politische Formation auf die Integration und das Management gesellschaftlicher Heterogenität verstand. Tief verankert im historisch gewachsenen sozialmoralischen Milieu des Katholizismus vor allem im deutschen Westen und Südwesten, gelang es den Christdemokraten seit der zweiten Hälfte der fünfziger und sechziger Jahre immer mehr, sich zur interkonfessionellen Sammelpartei nahezu aller christlichen, konservativen und bürgerlichen Milieus und Mentalitäten innerhalb der bundesrepublikanischen Gesellschaft zu entwickeln. Angesichts der jahrhundertealten Feindschaft zwischen Katholiken und Protestanten war das alles andere als eine Selbstverständlichkeit.

Anderswo in Europa gerieten katholisch geprägte Parteien im Zuge der Erosion ihrer lebensweltlichen Milieuvoraussetzungen immer tiefer in den Niedergang. Die CDU dagegen vermochte es auf einzigartige Weise, gerade in dieser Periode fortschreitender Entkonfessionalisierung und Säkularisierung die Beschränkungen ihrer katholischen Herkunft hinter sich zu lassen. Dass die protestantisch

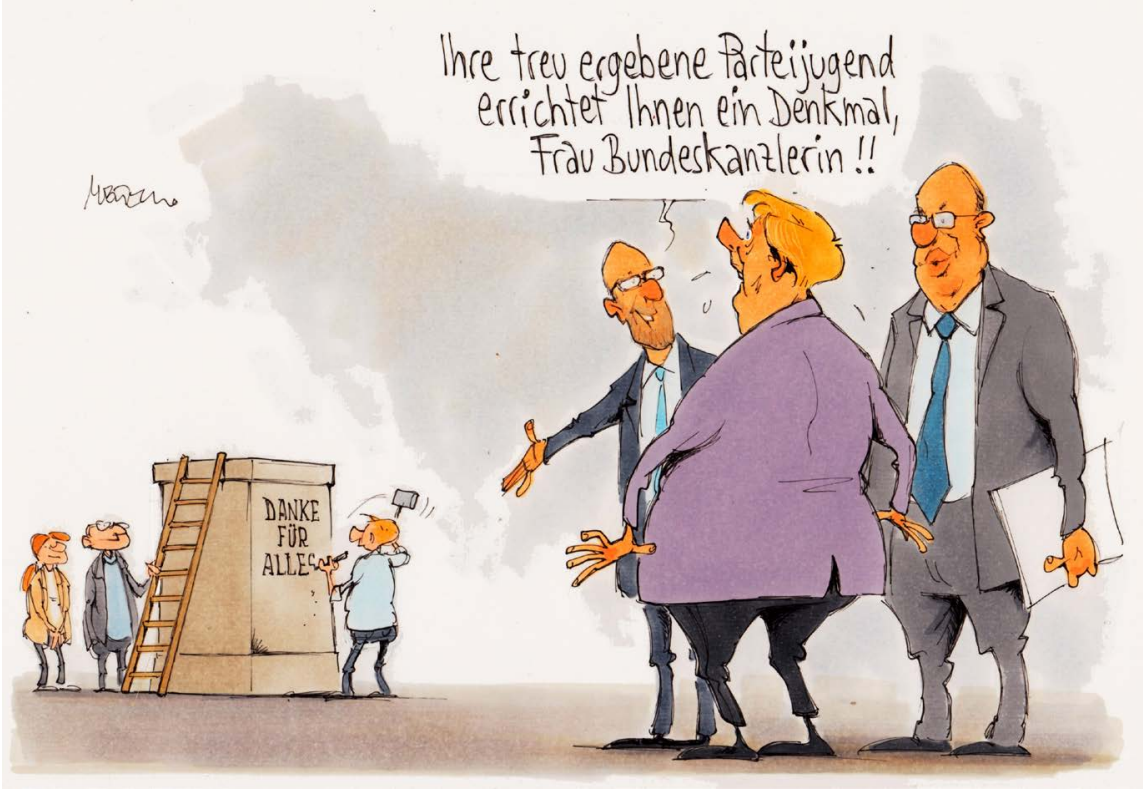


geprägten Bundesländer Schleswig-Holstein oder Niedersachsen in den siebziger und achtziger Jahren unangefochten von christdemokratischen Ministerpräsidenten geführt wurden, wäre noch zwei Jahrzehnte zuvor vollständig undenkbar gewesen. Der gemeinsame christliche Anspruch, Adenauers „hohes C“, wurde die Klammer, die zuvor unvereinbare konfessionelle Mentalitäten unter dem Dach der Christdemokratie zusammenführte. Und mindestens ebenso sehr trug gleichzeitig der starke Kitt des Antisozialismus dazu bei, die disparaten bürgerlichen und kleinbürgerlichen Teilmilieus unter dem breiten Dach der christdemokratischen Sammlungspartei zu integrieren.

Die Fähigkeit zur interkonfessionellen bürgerlichen Sammlung und der Antisozialismus - das waren die beiden zentralen Kraftquellen der Christdemokratie bei ihrem Aufstieg zur Hegemonialpartei der alten Bundesrepublik. Die CDU und ihre bodenständigen Lokaleliten verkörperten den Habitus von Maß und Mitte, in dem sich breite gesellschaftliche Schichten wiederfanden. Von den allerletzten Restbeständen dieser Kraftquellen in einstigen Hochburgen zehrt die Partei bis heute. Offensichtlich ist jedoch, wie dramatisch der Kurs dieser Ressourcen im Laufe der vergangenen Jahrzehnte abgestürzt ist: Die Fähigkeit zur interkonfessionellen Sammlung ist letztlich nur dort von Wert, wo Religion und Konfessionalität überhaupt noch eine gesellschaftsprägende Rolle spielen. Hingegen läuft der alte Appell an Bürgerlichkeit, Maß und Mitte ins Leere, wo über die Bedeutung solcher Kategorien keine auch nur einigermaßen geteilte Vorstellung mehr besteht. Und der Antisozialismus verliert seinen Sinn, wo sich irgendeine sozialistische Bedrohung einfach nicht mehr an die Wand malen lässt.

Im Deutschland des 21. Jahrhunderts ist die Christdemokratie so gesehen zu einer kulturell und programmatisch überständigen Partei geworden. Überzeugenden Ersatz für ihre verlorenen ideellen Ressourcen und ihre geschwundene gesellschaftliche Verankerung hat sie nicht gefunden. Bereits die gesamte „Ära Merkel“ von 2005 bis 2021 bedeutet genau besehen eben keine Wiederaufnahme und Fortsetzung des alten christdemokratischen Kontinuitätsfadens. Vielmehr müssen die vier Wahlperioden seit 2005 als ein einziger - ebenso unwahrscheinlicher wie paradoxer - Glücksfall für die deutsche Christdemokratie betrachtet werden: als geborgte Zeit, die ihr unvermittelt gerade in dem historischen Moment zufiel, als die früher entscheidenden Kraftquellen der Partei unwiederbringlich versiegt waren. Ja, die CDU hat seit 2005 erneut über viele Jahre hinweg das Kanzleramt besetzt gehalten. Eine Fortsetzung der vormaligen christdemokratischen Hegemonie bedeutete die Kanzlerschaft Angela Merkels aber allenfalls in dieser ganz formalen Hinsicht. Ein bisschen ähnelten die Christdemokraten in der Ära Merkel der legendären Comicfigur Wile E. Coyote, die, über den Klippenrand hinausgerannt, exakt so lange nicht in die Tiefe stürzt, wie sie noch nicht begriffen hat, dass sich unter ihr nur noch Luft befindet. 
Denn tatsächlich war der christdemokratische Kontinuitätsfaden gerissen, der selbstverständliche Einklang von Union und ideeller Gesamtmitte der Gesellschaft zerbrochen. In dieser Hinsicht war die Kanzlerschaft Merkel von Anfang an fragil und ohne gesellschaftliche Erdung. Bereits der unerhörte Aufstieg einer kompletten (nämlich erstens ostdeutschen, zweitens weiblichen und drittens protestantischen) Außenseiterin an die Spitze der deutschen Christdemokratie ließ sich überhaupt nur als Resultat und Ausdruck der schweren inneren Verstörung der Christdemokratie wie auch der fortgeschrittenen Entkopplung der Beziehung zwischen CDU und Gesellschaft begreifen. Ins Kanzleramt gewählt wurde Angela Merkel 2005 dann auch mit dem schlechtesten Resultat der Unionsparteien seit 1949 (35,2 Prozent) - ein Ergebnis, das die CDU unter Merkels Führung bei den Bundestagswahlen von 2009 (33,8 Prozent) und 2017 (32,9 Prozent) jeweils noch weiter unterbot.

Die ehrgeizige Außenseiterin Merkel hatte die CDU 1990 eher aus Zufall als Vehikel für ihre Karriere in der neuerdings gesamtdeutschen Politik entdeckt. Vermutlich allein das beherzte Eingreifen der politisch unbelasteten Generalsekretärin bewahrte die in der Spendenaffäre im Jahr 2000 existenziell angeschlagene CDU vor dem Untergang als Volkspartei. Merkel rettete die CDU in höchster Not, aber in der anschließenden „Ära Merkel“ gelang den Christdemokraten weder das Aufladen ihrer Sinnbestände, noch war im neuen, immer heterogeneren Deutschland auch nur ansatzweise eine Wiederkehr derjenigen alten gesellschaftlichen Verhältnisse möglich, in denen die Union zu Zeiten von Adenauer und Kohl als Dominanzpartei agiert hatte.

So erscheint die lange Ära Merkel im Rückblick als eine große Paradoxie. Ausgerechnet die Außenseiterin Merkel eröffnete der Christdemokratie noch einmal weitere 16 Jahre an der Macht - gerade zu einer Zeit, da dergleichen Machtausübung in Permanenz eigentlich völlig unwahrscheinlich geworden war. Christdemokratischen Nostalgikern und Traditionalisten hat es Merkel dennoch niemals recht machen können. Ihr Vorwurf lautet, Merkel habe - vermeintlich ohne Not - jahrein, jahraus den Ausverkauf des christdemokratischen „Tafelsilbers“ betrieben, dadurch das Profil der Partei immer weiter abgeschliffen und so überhaupt erst den Niedergang der Union heraufbeschworen. Dabei verkennen sie regelmäßig, dass die Voraussetzungen einer strukturellen Vorherrschaft der CDU in den Regierungsjahren Merkels von vornherein nicht mehr gegeben waren.

Als „Beweisstücke“ für Merkels Verrat an den Werten der CDU müssen immer wieder dieselben Umstände herhalten - vom Ausstieg aus der Kernenergie, über die Rettung Griechenlands in der Eurokrise, die Neuorientierung in der Familienpolitik und das Ende der Wehrpflicht bis hin zu den offenen Grenzen im europäischen Flüchtlingsjahr 2015. Dass für die CDU gleichzeitig auf der Ebene mancher Bundesländer Koalitionen ausgerechnet mit den Grünen machtpolitisch unab- 
wendbar geworden sind, tut ein Übriges, um die innere Demoralisierung der Partei voranzutreiben.

Zusammengefasst werden diese Tendenzen mit Vorliebe in der abfällig gemeinten Chiffre von der „Sozialdemokratisierung der CDU“. Diese Formel greifen zwar auch manche in der SPD immer wieder dankbar auf, um den Niedergang ihrer eigenen Partei als Ergebnis besonders perfider christdemokratischer Produktpiraterie zu erklären. Doch in Wirklichkeit hat es sich bei der Angela Merkel hier zugeschriebenen „Gesamtstrategie“ nie um mehr gehandelt als um eine beim ewigen „Fahren auf Sicht“ (Merkel) situativ entstandene Abfolge improvisierter Schritte zum Zweck der Machterhaltung. Etwas anderes als ein ,prozedural verengter Konservatismus“ (Thomas Biebricher) dieser Art war für der CDU in Wirklichkeit gar nicht mehr möglich, nachdem der alte Kitt christdemokratischer Identität, Mentalität und milieugebundener Sozialmoral zerbröselt war.

Gewiss hatte Angela Merkel ohnehin nie viel übrig für die normativen und kulturellen Traditionsbestände ihrer rheinisch-katholisch-westdeutsch geprägten Partei. Jedes tümelnde Schwelgen in christdemokratischer Traditionspflege und mutmaßlichen „konservativen Kernbeständen“ war ihr wesensfremd. Das alles ist schon aus biografischen Gründen nicht ihre Welt. Anders als ihre der Vergangenheit verhafteten Kritiker erfasste sie aber auch analytisch, dass sich christdemokratisches Regieren nicht mehr auf die untergegangenen Ressourcen gefestigter sozialmoralischer Milieus und ihrer Sinnbestände stützen konnte. Auf einem anderen Blatt steht, dass sie nie auch nur den Versuch unternahm, das entstandene Vakuum zu füllen. Irgendeinen revitalisierenden eigenen Beitrag gegen die inhaltliche Auszehrung, Sinnentleerung, „Erschöpfung“ und „Haltlosigkeit“ (Thomas Biebricher) der deutschen Christdemokratie hat Merkel in ihrer langjährigen Ägide als Generalsekretärin (1998-2000) und Vorsitzende (2000-2018) der CDU nicht auf den Weg gebracht. Auch für die künftige Ausrichtung der Partei, die sie fast zwei Jahrzehnte lang anführte, ohne sie zu prägen, schien sich Merkel am Ende kaum noch zu interessieren. Den in seiner ganzen Dynamik desaströsen Weg zur Auswahl von Armin Laschet zunächst als Parteivorsitzender, dann auch als Kanzlerkandidat, begleitete sie am Ende fast schon im Modus schicksalsergebener Resignation.

Mit Armin Laschet an der Spitze dürfte die deutsche Christdemokratie in den kommenden Monaten ihre nächste große Paradoxie-Erfahrung machen. In kultureller Hinsicht ist die Partei mit ihm gleichsam zu sich selbst zurückgekehrt. Der rheinische Katholik aus Aachen-Burscheid ist so tief verwurzelt im bürgerlichen und kleinbürgerlichen Restmilieu der altwestdeutschen CDU, wie es überhaupt nur möglich ist. Biografisch und habituell verkörpert Laschet in idealtypischer Weise alles, was seiner Partei an Angela Merkel immer fehlte - und notwendigerweise fehlen musste. Mit Laschet ist die Christdemokratie, verstanden als poli- 
tisches Soziobiotop, wieder ganz bei sich. Wer jedoch glaubt, die Partei werde, angeführt von Armin Laschet, inhaltlich und programmatisch zu schärferen Konturen zurückfinden, gar die vermeintliche „Sozialdemokratisierung“ der CDU in der Ära Merkel rückgängig machen, täuscht sich nicht nur im Charakter von Armin Laschet, sondern auch im Hinblick auf das Wesen der historischen CDU als immer etwas unbestimmter „Sammlungspartei“ der unterschiedlichsten Milieus, Positionen und Interessen. Genau diesen diffusen Wesenskern der deutschen Nachkriegschristdemokratie seit Adenauer verkörpert Armin Laschet mit seinem ewigen Mantra vom „Zusammenführen“ wie kein Zweiter. Doch Laschets Appell ist aus der Zeit gefallen und verklingt ins Leere. Denn was die CDU in früheren Zeiten trotz allem integrierte, ihr festes Korsett aus „hohem C“ und Antisozialismus sowie die Verankerung in intakten sozialmoralischen Milieus, spielt heute gesellschaftlich kaum noch eine Rolle.

Laschets Version des alten christdemokratischen Bewegungsprinzips „Integration zur Mitte hin“ wird daher bestenfalls zu vollständiger inhaltlicher Unschärfe und Beliebigkeit, schlimmstenfalls $\mathrm{zu}$ erbitterten innerparteilichen Kulturkämpfen führen. Die CDU war immer eine gut geölte Integrationsmaschine - und musste das aus machtpolitischen Gründen auch sein. Doch wo sich lebensweltlich und normativ bei bestem Willen kein kleinster gemeinsamer Nenner benennen lässt, wird das „Zusammenführen“ zur Absurdität. Eine CDU, in der obskure Verschwörungsideologen wie Hans-Georg Maaßen, die sektiererische „Werteunion“ sowie „nationalsozial“ orientierte ostdeutsche Landtagsfraktionen ebenso Platz finden sollen wie der Liberaldemokrat Ruprecht Polenz, der neoliberale Egozentriker Friedrich Merz oder die lesbisch-migrantische Nachwuchspolitikerin Diana Kinnert, kann notgedrungen nur als zusammenhangloses Nebeneinander von allen und allem aufrechterhalten werden.

Genau hierin besteht die neue Paradoxie. Armin Laschets milieugeerdeter rheinischer Radikalpragmatismus ohne inhaltliche Festlegung wäre in früheren Jahrzehnten das geeignete Rezept zur Führung einer binnenpluralen, aber in bestimmten Kernfragen eben doch einigen CDU gewesen. Angesichts der heutigen Haltlosigkeit einer ohne innere Mitte in viele Richtungen zugleich zerfasernden Partei aber kann Laschets Methode des beliebigen Sowohl-als-auch nur in die Sackgasse führen: Entweder die CDU verliert so noch weiter an Profil - und setzt ihren Sinkflug fort. Oder die Partei verstrickt sich in scharfe innerparteilicher Kultur- und Definitionskämpfe um den Charakter deutscher Christdemokratie unter den Bedingungen des 21. Jahrhunderts - und setzt ihren Sinkflug fort. Auch mit Kombinationen beider Zustände ist daher zu rechnen.

Der ostdeutschen CDU-Außenseiterin Angela Merkel haben innerparteiliche Kritiker^innen den typisch merkelschen Politikmodus der inhaltlichen Unschärfe immer zum Vorwurf gemacht. Als Kanzlerkandidat der Union treibt Armin Laschet 
diese Methode nun sogar noch weiter auf die Spitze. Gut 100 Tage vor der Bundestagswahl 2021 stand die Union als einzige Partei im Wettbewerb noch immer ohne Wahlprogramm da. Gleichzeitig schalteten die Christdemokraten Anzeigen in den sozialen Medien, in denen es hieß: „Welche Lösungsansätze hast Du für Deutschlands Zukunft? Bring Dich jetzt ein!“ Was werbewirksame die Möglichkeit von Partizipation simulieren sollte, brachte doch vor allem ein weiteres Mal eindringlich zum Vorschein, wie blank und ,inhaltsleer“ (Norbert Röttgen) die CDU nach 16 Jahren ununterbrochenen Regierens dasteht. Am Ende legte sie ein belangloses Wahlprogramm nach jahrzehntelang bewährtem Strickmuster auf den Tisch: „Manches muss sich ändern, doch die Kirche bleibt im Dorf.“

An sehr harte Grenzen stoßen wird die von Armin Laschet als Rückkehr zur bewährten Idee der christdemokratischen „Sammlungspartei“ missverstandene Beliebigkeit der CDU allerdings spätestens, sollte Laschet in die Lage geraten, als Bundeskanzler exekutive Verantwortung zu tragen. Dann nämlich müsste er bittere Pillen verschreiben, so oder so - und würde dafür kritisiert. Nicht zuletzt für ihren „radikal reaktiven Regierungsstil“ (Robin Alexander) wurde Merkel (nicht nur) aus ihrer eigenen Partei heraus regelmäßig angegriffen. Ihre Kritiker^innen verkannten, dass Merkels Methode für die CDU die einzig realistische Möglichkeit bedeutete, auch noch nach dem Verlust ihrer soziokulturellen und ideenpolitischen Voraussetzungen weiter Regierungsmacht auszuüben. In exakt derselben Lage würde sich ein Bundeskanzler Laschet wiederfinden. Der immer etwas zu wolkig daherredende Apologet des „Zusammenführens“ könnte nicht mehr immerzu händeringend ,abwägen“ (Laschet), sondern müsste vor allem auf klug strukturierte Weise Zumutungen verteilen.

Der „eigentliche ,Markenkern“ der CDU“, schrieb Thomas Biebricher vor dem Ausbruch der Corona-Epidemie, bestehe „mittlerweile in erster Linie darin, dass sie ein solides Management der Krisen und Verwerfungen einer scheinbar aus den Fugen geratenen Welt verbürgt“. Tatsächlich vermochte Angela Merkel vieles nicht - aber auf das „ruhige Abarbeiten der Problemberge und die Verzögerung allzu drastischer Transformationen" verstand sie sich in einzigartiger Weise. Dieser Merkel-Nimbus der CDU ist seit der Pandemie perdu. Aber davon abgesehen: Ist Armin Laschet in diesem Metier überhaupt der Richtige? Nur wenig spricht dafür. Und falls unerwartet doch: Wäre Deutschland angesichts der in den kommenden Jahren klimakrisenbedingt bevorstehenden großen Umbrüche nach diesem reaktiven Muster überhaupt noch effektiv zu regieren? Auch das ist äußerst unwahrscheinlich.

Als Bundeskanzlerin hat Angela Merkel der deutschen Christdemokratie 16 Jahre Aufschub an der Macht verschafft. Mehr nicht - aber das immerhin. Es könnte sogar sein, dass die CDU genau deshalb auch noch den nächsten Kanzler stellen wird. Das wäre der nächste Aufschub und erneut geborgte Zeit. Doch auf 
die Aufgaben, die nun folgen werden, sind weder die Christdemokrat*innen noch ihr Spitzenkandidat vorbereitet.

Dr. Tobias Dürr arbeitet als Politikwissenschaftler, Publizist, Buchautor, Redenschreiber und Politikberater.

\section{Literatur}

Alexander, Robin 2021: Machtverfall: Merkels Ende und das Drama der deutschen Politik. München.

Biebricher, Thomas: Geistig-moralische Wende: Die Erschöpfung des deutschen Konservatismus. Berlin.

Blasius, Moritz/Küpper, Moritz 2020: Der Machtmenschliche: Armin Laschet. Die Biografie, Essen: Klartext 2020

Dürr, Tobias/Walter, Franz 1999: Die Heimatlosigkeit der Macht: Wie die Politik in Deutschland ihren Boden verlor. Berlin.

Lucke, Albrecht von 2021: Verkehrte Welt: Grüne Eintracht, schwarzes Chaos. In: Blätter für deutsche und internationale Politik, Heft 5/2021, 5-8. 